\title{
Does ACS Management Differs in Different Region of Saudi Arabia
}

\section{Abstract}

Objectives: To compare the clinical features, management, and in-hospital outcomes of acute coronary syndrome (ACS) between patients in the Western region, non-invasive hospital (NIH) and other hospitals from Saudi Arabia that were involved in SPACE registry.

Methods: We compared NIH data; Seventy-one patients to 5055 patients enrolled in SPACE registry study for ST-elevation myocardial infarction (STEMI), and nonST elevation acute coronary syndrome (NSTEACS) including: non-ST elevation myocardial infarction and unstable angina.

Results: The mean age of the 71 patients tends to be higher in NIH (61.14 \pm $13.9)$ in comparison to the registry patients $(58.0 \pm 12.9$ years). No difference was noted in the male ratio; $69.7 \%$ vs. 77.4 , hypertension $67.6 \%$ vs. 55.3 and hyper lipidemia $52.1 \%$ vs. 41.4 . NIH patients had higher BMI of $28.03 \mathrm{~kg} / \mathrm{m}^{2}$ compared to 27.6 in the registry group. However, lower prevalence of DM was observed (45\% vs. $58.1 \%$ ) and proportion of smokers was $21.1 \%$ vs. 32.4 for NIH and registry groups respectively. Thirty-two percent of NIH patients had STEMI, in contrast to $41 \%$ in the registry and NIH had used ambulances more $(15.2 \%$ vs. $5.1 \%)$. In-hospital medications showed similar use of clopidogrel $(88.7 \%$ vs. 83.7 $\%$ ), no difference in the use of Aspirin (98.6\% vs. 97.7\%), angiotensin converting enzyme inhibitors/angiotensin receptor blockers (73.2\% vs. $75.1 \%)$, and statins (94.4\% vs. 93.3\%). However, beta-blockers use was significantly higher in the NIH group compared to the registry ( $91.5 \%$ vs. $81.6 \%, p=0.03$ ). In-hospital outcomes were not different in terms of recurrent myocardial infarction $(1.4 \%$ vs. $1.5 \%)$, cardiogenic shock ( $4.2 \%$ vs. $4.3 \%$ ), stroke ( 0 vs. $0.9 \%$ ), major bleeding ( $0 \%$ vs. $1.3)$. There was significant difference; in terms of recurrent ischemia $(19.7 \% \mathrm{vs}$. $12.6 \%, \mathrm{p}<0.05$ ) and In-hospital mortality (1.4 vs. $3.0 \%$ ).

Conclusion: ACS patients in NIH of KSA present at an older age, have similar prevalence of HTN and dyslipidemia, more access to ambulance use and higher recurrent ischemia compared with patients in the other regions of Saudi Arabia. There was appropriate use of evidence based guided therapy.

\section{Research Article}

Volume 4 Issue 1 - 2015

Abdulhalim Jamal Kinsara* and Jamilah Saad Alrahimi

Assistant Professor, Head of Adult Cardiology, King Saud bin Abdulaziz University for Health Sciences, Saudi Arabia

*Corresponding author: Abdulhalim Jamal Kinsara, Assistant Professor, Head of Adult Cardiology, King Saud bin Abdulaziz University for Health Sciences, King Faisal Cardiac Center, King Abdul Aziz Medical City- WR, Mail code 6599, P.O. Box 9515, Jeddah 21423, Saudi Arabia, Tel: 966-2-624 0000; ext. 25797; eFax 920008668; ext 25795;

Email: Kinsaraaj@ngha.med.sa

Received: October 15, 2015 | Published: November 27, 2015
Abbreviations: GRACE: Global Registry of Acute Coronary Events; SPACE: Saudi Project For Assessment of Coronary Events; STEMI: ST-Elevation Myocardial Infarction; NSTEACS: NonST Elevation Acute Coronary Syndrome; ACS: Acute Coronary Syndrome; NIH: Non-Invasive Hospital

\section{Introduction}

Ischemic heart disease represents a global health care burden, and is expected to increase in developing countries over the coming 10 years over $100 \%$ [1-5]. The presence of cardiovascular risk factors, alone or in combination, namely metabolic syndrome has high prevalence worldwide. Diabetes mellitus, hypertension, dyslipidemia and smoking play a major role in Saudi Arabia's population and influence the process of atherosclerosis and plaque formation at a young age. In the Saudi Arabian population in particular, the early detection, prevention and management of such risk factors and a knowledge of medical care are required to significantly decrease the burden of such diseases.

\section{Methods}

NIH data were analyzed using the data from The Saudi Project for Assessment of Coronary Events (SPACE) registry, a prospective registry of all consecutive ACS patients that were admitted to the participating hospitals. These include ST-elevation myocardial infarction (STEMI), and non-ST elevation acute coronary syndrome (NSTEACS): non-ST elevation myocardial infarction and unstable angina. Ethics committees at each hospital approved the study.

\section{Statistical analysis}

Data were presented as means \pm SD for the normally distributed data. The differences between groups were assessed using chi square or Fisher's exact test for categorical variables, student t-test for continuous variables for normally distributed variables, and the Mann-Whitney U test for skewed variables. All analyses were considered significant at $\mathrm{P}<0.05$.

\section{Results}

71 patient ACS patients were enrolled from our hospital, non invasive (NIH) that had no cardiac catheterization or cardiac surgery facilities. The mean age of the 71 patients was higher $(61.14 \pm 13.9)$ than the registry patients $(58.0 \pm 12.9$ years $)$. No 
difference was noted in the male ratio; $69.7 \%$ vs 77.4 , NIH patients had higher BMI of 28.03 compared to 27.6 in the registry group. Prevalence of hypertension in NIH was $67.6 \%$ vs $55.3,52.1 \%$ were hyperlipidemic vs.41.4. However, lower prevalence of DM was observed ( $45 \%$ vs. $58.1 \%$ ) and proportion of smokers was $21.1 \%$ vs. 32.4 for NIH and registry groups respectively (Table 1). Thirty-two percent of NIH patients had STEMI, while $41 \%$ in the registry. Access to ambulance was higher (15.2\% vs. 5.1\%) in NIH group. In-hospital medications showed use of clopidogrel (88.7\% vs. $83.7 \%$ ), no difference in the use of Aspirin (98.6\% vs. 97.7\%), angiotensin converting enzyme inhibitors/angiotensin receptor blockers $(73.2 \%$ vs. $75.1 \%)$, and statins $(94.4 \%$ vs. $93.3 \%)$.
However, beta-blockers use was significantly higher in the Jeddah group compared to the registry ( $91.5 \%$ vs. $81.6 \%, \mathrm{p}=0.03)$, may be due to higher contraindication to beta-blockers in the registry, Table 2.

In-hospital outcomes were not difference in term of recurrent myocardial infarction ( $1.4 \%$ vs. $1.5 \%)$, cardiogenic shock $(4.2 \%$ vs. $4.3 \%$ ), stroke ( 0 vs. $0.9 \%$ ), major bleeding ( $0 \%$ vs. 1.3 ). There was significant difference in terms of recurrent ischemia $(19.7 \%$ vs. $12.6 \%$; $\mathrm{p}<0.05)$ an advantage known for people who had coronary angiogram/PCI. In-hospital mortality was significantly lower in NIH, probably due to low prevalence of DM compared with the registry (1.4 vs. $3.0 \%$ ), Table 3.

Table 1: Baseline characteristics.

\begin{tabular}{|c|c|c|c|}
\hline & Overall (n=71) N (\%) & Overall (n=5055) N (\%) & P value \\
\hline Age & $61.14 \pm 13.95$ & $58.0 \pm 12.9$ & 0.0497 \\
\hline Sex: M & $51(71.8)$ & $3914(77.4)$ & 0.136 \\
\hline BMI (mean \pm SD) & $28.03 \pm 5.3$ & $27.6 \pm$ & \\
\hline Hypertension & $48(67.6)$ & $2783(55.3)$ & 0.04 \\
\hline History of CAD & $11(15.5)$ & $2145(42.4)$ & $<0.001$ \\
\hline History of Hyperlipidemia & $37(52.1)$ & $2084(41.4)$ & 0.07 \\
\hline New DM & $12(16.9)$ & $2937(58.1)$ & $<0.01$ \\
\hline Current smoking & $15(21.1)$ & $1638(32.4)$ & 0.04 \\
\hline Past CVS - CABG & $3(4.2)$ & $295(5.8)$ & 0.57 \\
\hline Past CVS - CVATIA & $6(8.5)$ & $309(6.1)$ & 0.04 \\
\hline Family history of premature CAD & $9(13.6)$ & $719(15.4)$ & 0.687 \\
\hline SBP $<90$ & $1(1.4)$ & $148(3.2)$ & 0.8 \\
\hline Killip class>1 & $43(60.6)$ & $940(20.4)$ & $<0.001$ \\
\hline HR>100 & $16(22.5)$ & $678(14.8)$ & 0.07 \\
\hline Ambulance use & $11(15.2)$ & $186(5.1)$ & 0.003 \\
\hline
\end{tabular}

Table 2: In-hospital treatments.

\begin{tabular}{|c|c|c|c|}
\hline & Overall $(\mathbf{n = 7 1 )}$ & Overall $(\mathbf{n = 5 0 5 5 )}$ & P value \\
\hline Aspirin at discharge & $70(98.6)$ & $4939(97.7)$ & 0.21 \\
\hline Clopidogrel & $63(88.7)$ & $4227(83.7)$ & 0.26 \\
\hline Beta blocker & $65(91.5)$ & $4118(81.6)$ & 0.03 \\
\hline ACE/ ARB & $52(73.2)$ & $3801(75.1)$ & 0.71 \\
\hline Statins & $67(94.4)$ & $4707(93.3)$ & 0.71 \\
\hline
\end{tabular}

Table 3: In-Hospital Outcomes.

\begin{tabular}{|c|c|c|c|}
\hline & Overall(n=71) & Overall(n=5055) & P value \\
\hline Death & $1(1.4)$ & $154(3.0)$ & 0.001 \\
\hline Recurrent MI & $1(1.4)$ & $77(1.5)$ & 0.45 \\
\hline Recurrent Ischemia & $14(19.7)$ & $638(12.6)$ & 0.03 \\
\hline Congestive heart failure & $5(7.0)$ & $519(10.2)$ & 0.47 \\
\hline Cardiogenic shock & $3(4.2)$ & $220(4.3)$ & 0.93 \\
\hline Stroke & 0 & $43(0.9)$ & 0.44 \\
\hline Major Bleeding & 0 & $68(1.3)$ & 0.51 \\
\hline
\end{tabular}




\section{Discussion}

This study aimed to compare the clinical features, management and In-Hospital outcomes of acute coronary syndrome between patients at tertiary NIH hospital, serving around 3 million people and other several hospitals from all geographic regions of Saudi Arabia that were involved in SPACE registry but had invasive facility. It provides a good representation and comparison of the acute coronary syndrome clinical data since few data from the Arabian and Middle East area have been published.

Of note the whole population were younger than the international data. Old age (above 65 years) is an independent factor for the outcome in acute coronary syndrome; it is part of the risk assessment score (TIMI) for mortality and morbidity assessment and was found to be an independent prognostic predictor of outcomes in the Global Registry of Acute Coronary Events (GRACE) registry [6]. The percentages of cardiovascular risk factors including hypertension, dyslipidemia and smoking showed minimal difference between the two groups. The NIH patients had higher BMI of 28.03 however lower prevalence of DM was observed, in contrast to previously published data. This, in part, could be due to small sample size. Seventy-one patients were enrolled in NIH hospital showing high prevalence of metabolic syndrome. The different demographic outcomes had many similarities in the two groups. These data are comparable to previous findings reported higher evidence of metabolic syndrome leading to ACS in the Saudi population [7-9].

More cases of STEMI were noted in the registry but there was more utilization of out-hospital medical access in NIH compared to other regions in the kingdom. The geographical area and the health care system. Plays major roles in easy access to medical care and such findings should highlight an important element that improves this outcome, particularly door to needle or door to balloon. Maintenance of evidence-based guide therapy our data showed no significant difference between both groups with use of In-hospital medications including clopidogrel, Aspirin, angiotensin converting enzyme inhibitors/angiotensin receptor blockers, and statins.

Several observational studies showed similar results to our findings with no statistical difference in the In-hospital outcome between ACS patients treated medically which include recurrent myocardial infarction, cardiogenic shock and major bleeding [9-11]. Our findings paralleled those reported in the COURGE trial showing that despite having no interventional therapy with coronary revascularization either PCI or bypass surgery in NIH hospital, optimum medical therapy for coronary artery disease, patients showed no major difference in developing cardiovascular events [12]. There was a significant difference in terms of recurrent ischemia, however, that was not reflected in total In-hospital mortality, despite medical management being the only option for patients treated in $\mathrm{NIH}$. These findings lend further support that continuing management with anti ischemic medication in particular statin and antiplatelet in addition to blood sugar and blood pressure control, significantly decreases the In-hospital morbidity and mortality $[11,12]$. We recognize that the sample is not large enough but our study will give insight to the magnitude of the problem.

\section{Conclusion}

NIH results as compared to the SPACE registry showed that our ACS patients have similar prevalence of cardiovascular risk factors and In-hospital outcome with appropriate use of evidenceguided medical therapy.

\section{References}

1. Aljefree N, Ahmed F (2015) Prevalence of Cardiovascular Disease and Associated Risk Factors among Adult Population in the Gulf Region: A Systematic Review. Advances in Public Health 23.

2. Murray CJ, Vos T, Lozano R, Naghavi M, Flaxman AD, et al. (2012) Disability-adjusted life years (DALYs) for 291 diseases and injuries in 21 regions, 1990-2010: a systematic analysis for the Global Burden of Disease Study 2010. Lancet 380(9859): 2197-223.

3. Lopez AD, Mathers CD, Ezzati M (2006) Global and regional burden of disease and risk factors, 2001: systematic analysis of population health data. Lancet 367(9524): 1747-1757.

4. Yusuf S, Reddy S, Ounpuu S (2001) Global burden of cardiovascular diseases: Part I: general considerations, the epidemiologic transition, risk factors, and impact of urbanization. Circulation 104(22): 27462753.

5. Reddy KS (2004) Cardiovascular disease in non-Western countries. N Engl J Med 350: 2438-2440.

6. Steg PG, Goldberg RJ, Gore JM (2002) Baseline characteristics, management practices, and in-hospital outcomes of patients hospitalized with acute coronary syndromes in the Global Registry of Acute Coronary Events (GRACE). Am J Cardiol 90(4): 358-363.

7. Kumosani TA, Alama MN, Iyer A (2011) Cardiovascular diseases in Saudi Arabia. Prime Res Med (PROM) 1: 1-6.

8. Al-Nozha MM, Arafah MR, Al-Mazrou YY, Al-Maatouq MA, Khan NB, et al. (2004) Coronary artery disease in Saudi Arabia. Saudi Med J 25(9): 1165-1171.

9. Osman AK, Al-Nozha MM (2000) Risk factors of coronary artery disease in different regions of Saudi Arabia. East Mediterr Health J 6(2-3): 465-474.

10. Rogers WJ, Canto JG, Barron HV, Boscarino JA, Shoultz DA, et al. (2000) Treatment and outcome of myocardial infarction in hospitals with and without invasive capability. Investigatorsin the National Registry of Myocardial Infarction. J Am Coll Cardiol 35(2): 371-379.

11. Rosengren A, Wallentin L, Simoons M, Gitt AK, Behar S, et al. (2006) Age, clinical presentation, and outcome of acute coronary syndromes in the EuroHeart acute coronary syndrome survey. Eur Heart J 27(7): 789-795.

12. Boden WE, O’Rourke RA, Teo KK, Har- tigan PM, Maron DJ, et al. (2007) Optimal medical therapy with or without PCI for stable coronary disease. N Engl J Med 356(15): 1503-1516. 